OPEN ACCESS

Edited by:

Lei Ye,

National Heart Centre Singapore,

Singapore

Reviewed by:

Wendy Keung,

The University of Hong Kong,

Hong Kong

Jianhua Zhang,

University of Wisconsin-Madison,

United States

*Correspondence:

Bingying Zhou

zhoubingying@fuwaihospital.org

${ }^{\dagger}$ These authors have contributed equally to this work

Specialty section:

This article was submitted to

Stem Cell Research,

a section of the journal

Frontiers in Cell and Developmental

Biology

Received: 18 January 2021

Accepted: 11 March 2021

Published: 01 April 2021

Citation:

Huang J, Feng $Q$, Wang $L$ and

Zhou B (2021) Human Pluripotent

Stem Cell-Derived Cardiac Cells: Application in Disease Modeling, Cell

Therapy, and Drug Discovery.

Front. Cell Dev. Biol. 9:655161. doi: 10.3389/fcell.2021.655161

\section{Human Pluripotent Stem Cell-Derived Cardiac Cells: Application in Disease Modeling, Cell Therapy, and Drug Discovery}

\author{
Juan Huang1,2†, Qi Feng't, Li Wang ${ }^{1,2}$ and Bingying Zhou ${ }^{2 *}$ \\ ${ }^{1}$ Shenzhen Key Laboratory of Cardiovascular Disease, Fuwai Hospital, Chinese Academy of Medical Sciences, Shenzhen, \\ China, ${ }^{2}$ State Key Laboratory of Cardiovascular Disease, Fuwai Hospital, National Center for Cardiovascular Diseases, \\ Chinese Academy of Medical Sciences and Peking Union Medical College, Beijing, China
}

Cardiac diseases are the leading cause of deaths worldwide; however, to date, there has been limited progress in the development of therapeutic options for these conditions. Animal models have been the most extensively studied methods to recapitulate a wide variety of cardiac diseases, but these models exhibit species-specific differences in physiology, metabolism and genetics, which lead to inaccurate and unpredictable drug safety and efficacy results, resulting in drug attrition. The development of human pluripotent stem cell (hPSC) technology in theory guarantees an unlimited source of human cardiac cells. These hPSC-derived cells are not only well suited for traditional two-dimensional (2-D) monoculture, but also applicable to more complex systems, such as three-dimensional (3-D) organoids, tissue engineering and heart on-a-chip. In this review, we discuss the application of hPSCs in heart disease modeling, cell therapy, and next-generation drug discovery. While the hPSC-related technologies still require optimization, their advances hold promise for revolutionizing cell-based therapies and drug discovery.

Keywords: pluripotent stem cell, cell therapy, drug discovery, cardiac cell, disease modeling

\section{INTRODUCTION}

Although cardiac diseases, such as myocardial infarction and heart failure, have been the leading cause of deaths worldwide, few drugs are being approved yearly compared with many other disease area, resulting in a huge gap between clinical need and drug development (Fordyce et al., 2015). The regenerative capacity of the heart is quickly lost during mammalian postnatal development. Pathological insults, such as ischemia, almost invariably lead to irreversible cardiac cell loss, which poses the greatest challenge to the treatment of cardiac diseases. In the past decades, rapid progress in human pluripotent stem cell (hPSC) technology enabled the generation of major cardiac cell types, thus unlocking new possibilities to treat patients with the most debilitating forms of heart disease using cell-based therapies (Mummery et al., 2012; Protze et al., 2019; Williams and Wu, 2019). To date, several studies have shown that transplantation of hPSC-derived cardiomyocytes holds great promise for attenuating cardiac dysfunction and reducing consequent fibrotic scarring (Gao et al., 2018; Liu et al., 2018). 
Animal models are arguably the most widely used method to model disease onset and progression, providing valuable mechanistic insights in an in vivo setting. However, the extrapolation of translatable data from animals data to guide the treatment of human heart disease has been difficult due to considerable species differences (Matsa et al., 2014). hPSCs can potentially bridge this transitional gap by providing an unlimited source of human cardiac cells for biomedical research and drug discovery (Matsa et al., 2014). Protocols that enable the generation of cardiomyocyte subtypes such as ventricular, atrial, and sinoatrial pacemaker cells, as well as non-myocyte cell types including endothelial cells and fibroblasts have been developed (Mummery et al., 2012; Weng et al., 2014; Lee et al., 2017; Protze et al., 2017; Williams and Wu, 2019; Zhang et al., 2019). These highly enriched populations of specific cardiomyocyte subtypes facilitate disease modeling and drug testing by recognizing cellular, molecular and functional heterogeneity within the heart. Further, considering the spatial complexity of the heart, hPSC-based 3-D multicellular systems, including human heart organoids (Mills et al., 2017; Giacomelli et al., 2020), engineered heart tissues (EHTs) (Mannhardt et al., 2016), and heart-onchip models (Zhang et al., 2016) have been shown to more accurately predict human cardiac biology and pathophysiology (Sharma et al., 2020). Incorporation of electrophysiology, epigenomics, transcriptomics, proteomics, metabolomics and imaging techniques will equip these hPSC-based platforms with additional tools to accomplish patient-specific disease modeling and personalized drug response screening.

In this review, we summarize the current progress of the application of hPSCs in cardiac disease and cell therapies (Figure 1). We also highlight the application of available hPSC platforms for the pharmacologic evaluation and cardiac safety assessment of drugs (Figure 1).

\section{CARDIAC DISEASE MODELING USING hPSCs}

Modeling heart disease is central to the understanding of the pathological processes. Conventional models of cardiovascular disease rely mostly on animals. However, the inherent differences between species render elucidation of human heart pathology unexpectedly difficult (Savoji et al., 2019). Therapeutics with promising results in animals often failed to show any improvement in clinical trials. Therefore, human-derived disease models bear the unique advantage to more faithfully represent human disease, thus potentially providing a more refined comprehension of disease mechanisms, paving the way to new therapeutic options (Zhao et al., 2020).

One of the major causes of cardiac disorders is genetic mutations (Dell'Era et al., 2015). The advent of iPSC technology provides a powerful means to model genetic cardiac diseases by using somatic cells directly from patients. In the past decade, various genetic cardiac disorders have been successfully modeled using iPSCs. For example, Timothy syndrome is caused by a missense mutation in L-type calcium channel $\mathrm{Ca}_{v} 1.2$ that leads to excess $\mathrm{Ca}^{2+}$ influx, prolonged action potential and irregular contraction. Yazawa et al. (2011) reported the generation of iPSC-CMs derived from patients with Timothy syndrome, and these cells demonstrated irregular electrical activity and abnormal $\mathrm{Ca}^{2+}$ signaling similar to the cardiac phenotype found in the patients. iPSCs have also been used to model LEOPARD syndromes with PTPN11 gene mutation (Carvajal-Vergara et al., 2010), long QT syndromes with a mutation in KCNQ1 (Friedrichs et al., 2013), arrhythmogenic right ventricular dysplasia with PKP2 mutations (Kim et al., 2013), and dilated cardiomyopathy with TTN mutations (Hinson et al., 2015).

One potential drawback of such application is that differences in the genetic background among cell lines may conceal the true phenotype induced by a single mutation. To address this challenge, the introduction of genome-editing techniques, such as clustered regularly interspaced short palindromic repeats(CRISPR-) associated protein 9 (Cas9) system, help to generate isogenic iPSC lines, and allows researchers to study the precise effect of a mutation on the onset or progression of cardiac diseases while avoiding confounding genetic factors (Seeger et al., 2017; Lam and Wu, 2018). By correcting mutations or insertions, CRISPR/Cas9 further facilitates the elucidation of the causal role of the mutation. For example, CRISPR/Cas9 was successfully used to identify SCN5A as a causative mutation of arrhythmogenic right ventricular cardiomyopathy (ARVC), and corrected cells showed normal channel activity (Te Riele et al., 2017).

The heart is composed of multiple cell types, including cardiomyocytes and non-cardiomyocytes, and the intricate crosstalk and multifaceted regulation among cells are central to heart homeostasis and disease. In this perspective, co-culture of hiPSC-derivatives, 3-D organoids, EHTs and microfluidic organ-chips exhibit distinct advantages over mono-lineage cultures (Figure 2). Using relatively immature iPSC-CMs to represent adult-onset disorders remains challenging. Recent studies demonstrated that co-culture of fibroblasts, epicardial cells, or endothelial cells with hiPSC-CMs enhanced the maturity of hiPSC-CMs, which improved modeling of cardiac diseases (Bargehr et al., 2019; Giacomelli et al., 2020). Still, there are some limitations regarding regular 2-D culture, such as lacking a 3D extracellular matrix and a defined auxotonic load. 3$\mathrm{D}$ cardiac constructs including organoids and EHTs overcome these limitations and improve representation of the in vivo cardiovascular environment. Cardiac organoids are generated from hPSCs that self-assemble and -organize into complex native-like organ structures (Richards et al., 2020). Voges et al. (2017) reported successful generation of an AMI model in human ESC-derived cardiac organoids. Cryoinjury induced local tissue damage, while the adjacent cells remained viable. Functional evaluation showed that tissue regeneration accompanied by functional recovery was seen 14-days post-injury (Voges et al., 2017). Another hiPSC-derived cardiac organoids that cooperate an oxygen-diffusion gradient and that are stimulated with neurotransmitter noradrenaline was shown to recapitulate the infarcted, border and remote zones of myocardial infarction in the human heart, with concurrent modeling of the hallmarks of AMI, including metabolic shifts, fibrosis and aberrant calcium handling (Richards et al., 2020). 


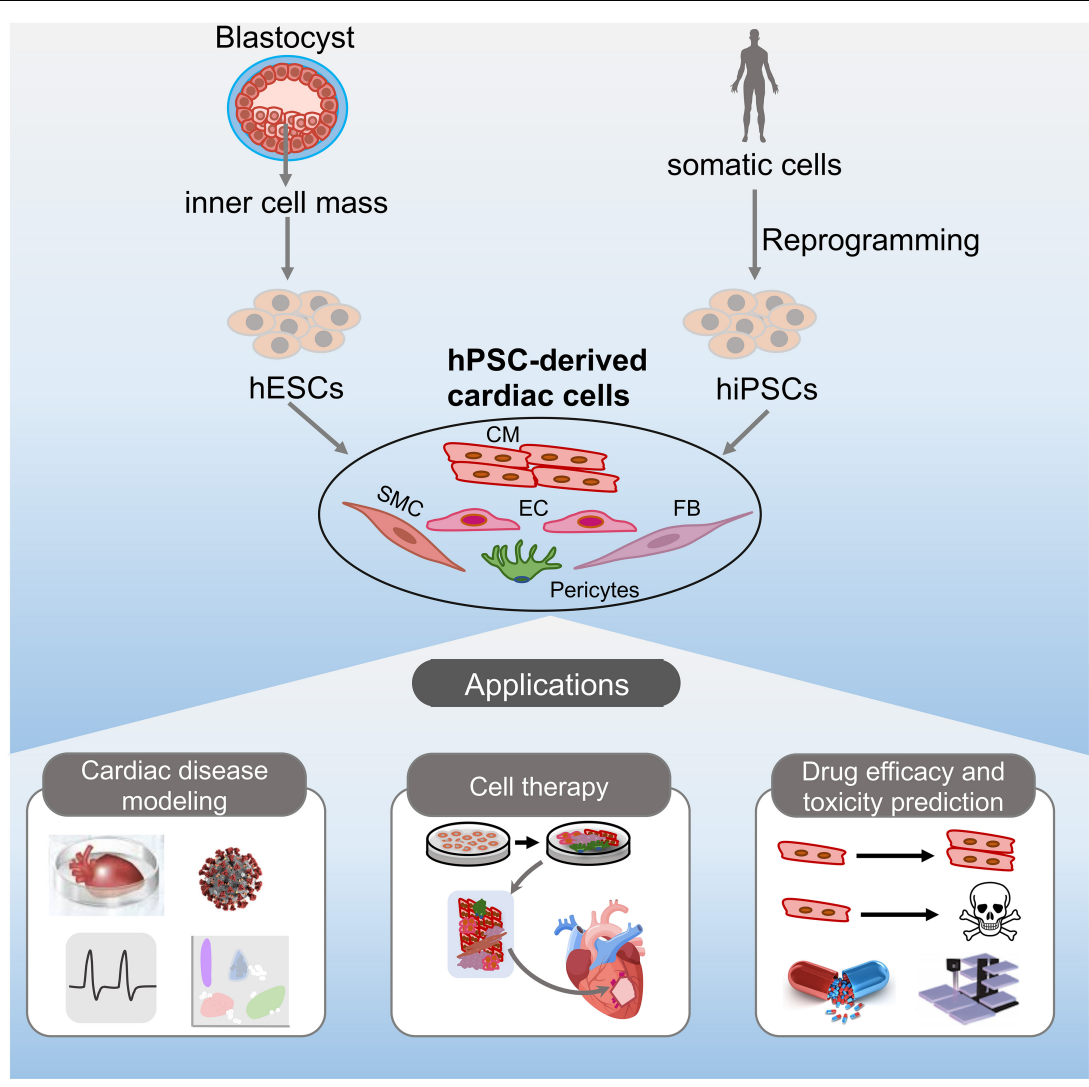

FIGURE 1 | Potential applications of hPSC-derived cardiac cells. hESCs or hiPSCs can differentiate into various cardiac cell types in vitro, including cardiomyocytes, endothelial cells, fibroblasts, smooth muscle cells, and pericytes. The resulting cells can be potentially used for disease modeling, cell therapy, and drug discovery.

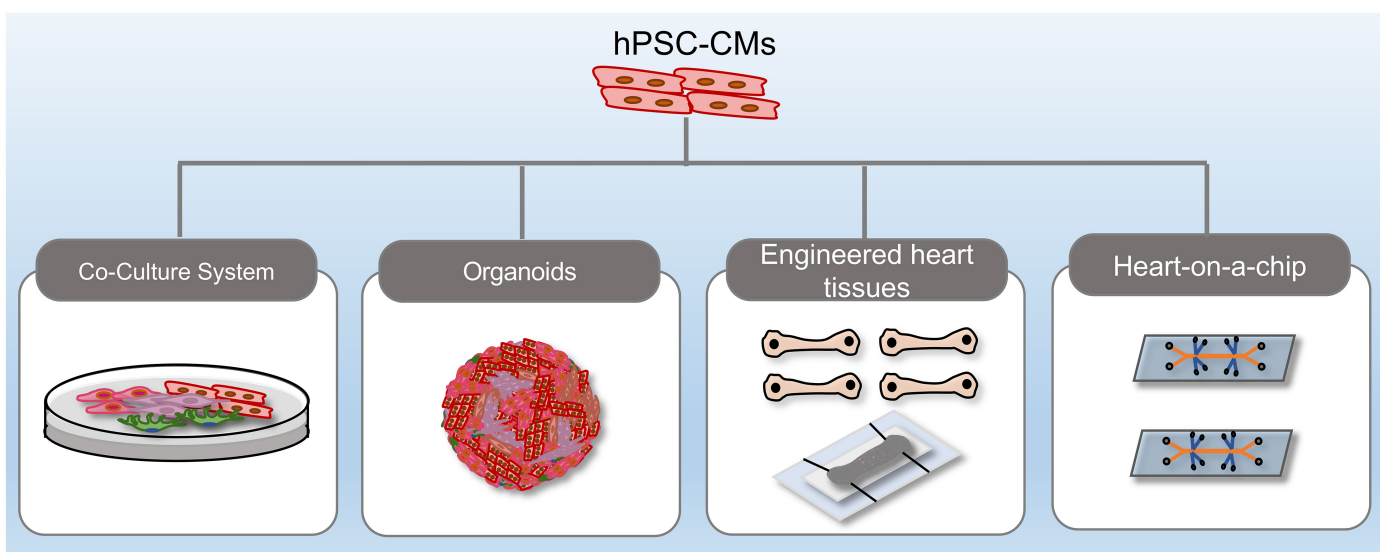

FIGURE 2 | Recent methodological advances in hPSC-derived complex platforms. hPSC-derived platforms have grown in complexity from simple, two-dimensional cultures into multi-lineage co-cultures, heart organoids, engineered heart tissues, and heart-on-a-chip systems.

Engineered heart tissues can be generated by mixing hPSCderived cardiomyocytes with extracurricular matrix components such as fibrinogen, collagen or Matrigel (Breckwoldt et al., 2017). The generation of EHTs also requires a casting mold that determines the 3-D shape of the heart tissue, and a support structure that provides mechanical restraint of the developing heart tissue (Eder et al., 2016). EHTs are well suited to evaluate the effects of mechanical stimulation, and therefore can mimic afterload enhancement-induced disease conditions such as aortic valve stenosis, chronic hypertension, and cardiac hypertrophy (Hirt et al., 2012; Tzatzalos et al., 2016). Since EHTs are little heart muscles, they allow measurements of all essential parameters of 
heart muscle function, including, but not restricted to, contractile force, conduction velocity, beating rate, rhythm, diastolic tension, passive tension, and intracellular $\mathrm{Ca}^{2+}$ transients (Hirt et al., 2014). Besides, EHTs are also compatible with histological analyses, protein detection, and sequencing techniques, such as single-cell RNA-sequencing.

The development of 3D stamping and bioprinting techniques provides scaffolds to generate heart-on-a-chip. EHTs and hearton-a-chip methods allowed modeling of specific cardiac diseases, including Barth-syndrome-associated cardiomyopathy (Wang et al., 2014), Duchenne muscular dystrophy (Long et al., 2018) and primary hypertension-induced left ventricular hypertrophy (Zhao et al., 2019). Zhao et al. (2019) took advantage of organ-on-a-chip engineering and organoid self-assembly to generate mature ventricular tissue, and to perform electrical conditioning for up to 8 months, allowing modeling of chronic, polygenic conditions. With the generation of a wide variety of human organ-on-a-chip models, great efforts have been made to integrate multisensory systems. Zhang et al. (2017) reported two multiorgan models, liver- and-heart-on-a-chip and heartliver-cancer-on-a-chip, for automated and continual in situ monitoring of organoid behaviors.

Recently, hPSCs have also been proven quite useful in the study of COVID-19-related heart disorders. To date, this pandemic has led to more than 121 million infections and 2.6 million deaths worldwide. Apart from respiratory complications, COVID-19 is also known to induce cardiac complications, including myocardial injury, arrhythmias, acute coronary syndrome, which are major indicators of poor prognosis (Nishiga et al., 2020; Zheng et al., 2020). Since one of the major receptors for COVID-19 is not recognized by the virus in mice, hPSCs were rapidly recognized as an invaluable tool to address this problem, and has already yielded important insight into virushost interactions, immune responses and the cytokine storm (Yang et al., 2020; Yiangou et al., 2020). Combined with various biochemical, cellular, molecular and genetic studies, these newly developed hPSCs-cardiac models will likely contribute much more to uncovering key mechanisms and therapeutic strategies of COVID-19-induced cardiac damage (Yiangou et al., 2020).

\section{hPSC-BASED CELL THERAPY}

Aside from mechanistic studies of cardiac disease, replenishing damaged myocardium with healthy cells (i.e., cell therapy) was another hotly pursued area of application. Transplantation of hESC-derived cardiomyocytes (hESC-CMs) was found to improve cardiac function in postinfarct rats and pigs (Kehat et al., 2004; Caspi et al., 2007; Laflamme et al., 2007). More importantly, Chong et al. (2014) showed that exogenously transplanted hESC-CMs remuscularized the infarcted area in a non-human primate model. However, hESCs may not be the best option for clinical treatment due to ethical concerns and immune rejection. Ethical concerns arise from their origins, since these cells are isolated from the inner cell mass of the human embryo, leading to the destruction of the latter. Immune rejection occurs due to allogenic transplantation of cells. By contrast, iPSC technology, in which pluripotent stem cells are directly reprogrammed from the same patient's somatic cells, effectively circumvents the ethical issues associated with the use of ESCs. Additionally, iPSCs are considered autologous, and are believed not to require immunosuppression. Gene editing in hiPSC further helps to reverse disease phenotype by correction of the pathogenic mutation or variant, raising the possibility of personalized therapies for autologous stem cell transplantation.

Injecting hPSC-derived cardiac cells into the myocardium to replace dead cells appears to be a very straightforward concept. However, in practical terms, controlling the exact cell number, limited efficiency in cell delivery to target sites, and batch differences, all pose great challenges to the application of hPSC-based cell therapy (Matsuura et al., 2013; Guo et al., 2020). With the development of tissue engineering, stem cellderived cell sheets gradually showed their superiority over direct injection in heart tissue repair (Matsuura et al., 2013). Kawamura et al. (2012) showed that highly pure (almost 90\%) hiPSCCMs sheets were able to attenuate left ventricular remodeling, increase neovascularization as well as inhibit fibrosis 8 weeks after cell transplantation into 12 -week porcine models of myocardial infarction. The same group demonstrated that the combined use of cell sheets and the omental flap technique was beneficial even in treating severe heart failure (Kawamura et al., 2017). Most recently, it was reported that the transplantation of clinically relevant dimensions $(4 \mathrm{~cm} \times 2 \mathrm{~cm} \times 1.25 \mathrm{~mm})$ of human cardiac muscle patches significantly improved left ventricular function and reduced infarct size in infarcted swine (Gao et al., 2018).

Mechanistically, transplantation of hiPSCs-derived cardiac cells may provide a favorable microenvironment for pre-existing cells in the infarcted zone to proliferate, thus preventing serious post-MI events (Ye et al., 2014). The mechanisms by which injected cells exert these effects are still not fully clear. It has been shown that only a limited number of transplanted cells can differentiate and retain in the host myocardium after delivery, suggesting that the beneficial effects of cell therapy are mediated by the activation of paracrine pathways which leads to endogenous regeneration (Tachibana et al., 2017). The autocrine and paracrine factors facilitate angiogenesis, promote vascularization, attenuate fibrosis and relieve inflammation. Still, the clinical translation of hPSC-based cell therapy is facing a major problem, i.e., the immaturity of hPSC-derived cells, which can cause life-threatening arrhythmia, and teratoma formation (Cambria et al., 2017). Even vascularized cell sheets co-cultured with different types of cells exhibit an immature phenotype. As a source of de novo cardiomyocytes, hPSC-CMs have so far yielded only a short-term improvement in cardiac function ranging from weeks to months. Therefore, more advanced strategies to induce maturation, vascularization, and to improve durable cardiac function recovery are worth investigation.

\section{hPSCs-BASED DRUG DISCOVERY}

Human pluripotent stem cell technology is also widely used in cardiovascular drug discovery, providing pharmacologic and toxicologic predictions. Yazawa et al. (2011) found 
that roscovitine, a compound that increases the voltagedependent inactivation of $\mathrm{CaV} 1.2$, rescued the cardiomyocyte phenotypes in Timothy syndrome. Using hPSC-derived cardiomyocytes and organoids, compounds that promoted human heart muscle cell proliferation, but minimally affected heart rhythm and contractility, were identified (Mills et al., 2019). Although the identified compounds displayed poor pharmacokinetic properties, and were only maintained at pro-proliferative doses in vivo for a short period, they led to the elucidation of mevalonate pathway important for cardiomyocyte proliferation. Most recently, Theodoris et al. (2020) reported that a network-based screen in iPSCderived cells revealed therapeutic candidates for a common form of heart disease involving the aortic valves, suggesting that the combined use of network-based screening, iPSC technique, and machine learning may represent an effective approach for drug discovery. Together, these inspiring hPSCbased drug studies offer meaningful pipelines to identify drug candidates that may lead to new therapeutic options for heart disease.

Unexpected cardiotoxicity is a major cause of drug attrition and drug withdrawal from the market (Laverty et al., 2011). Traditional methods of preclinical cardiac safety evaluation mainly rely on animal models, which tend to be expensive, low-throughput, and exhibit species differences in cardiac physiology (Mercola et al., 2013). Alternative methods to identify cardiotoxic drugs involve the heterologous expression of cardiac ion channels in non-cardiac cells. However, these non-cardiac cells lack CM-specific structural components such as sarcomeres, and inhibition of specific ionic currents alone cannot accurately measure the effects of drug candidates, which could miss potential arrhythmogenic effects or generate falsepositive readings. Other cells, such as healthy and primary human CMs, may be used for preclinical evaluation of drug cardiotoxicity. However, sample access and cellular abundance may be prohibitive for its use in an industrial setting. Regulatory agencies around the globe, including US FDA, European EMA, Health Canada, and Japan NIHS, have recognized the lack of standardized assessment of cardiac safety, and thus encouraged the development of the Comprehensive In Vitro Proarrhythmia Assay (CiPA), which aims to explore the utility of hPSC-derived cardiomyocyte assays in evaluating cardiac safety and arrhythmogenesis (Sager et al., 2014). Data from recent studies support the utility of hPSC-derived cardiomyocytes for predicting drug-induced arrhythmia (Sager et al., 2014; Gintant et al., 2016; Blinova et al., 2018). Besides electrophysiology, hiPSC-CMs are also used to measure drug-induced alterations in cellular contractility and viability, thus broadening the scope of cardiac safety evaluation. Sharma et al. (2018) developed a detailed methodology to generate hiPSC-CMs and subsequently use these cells to evaluate drug-induced cardiotoxicity by using contractility and cytotoxicity assays. Using this platform, they performed cardiotoxicity screening of tyrosine kinase inhibitors, with results correlating with clinical phenotypes (Sharma et al., 2017). They also demonstrated that the observed toxicities could be ameliorated with cardioprotective insulin/IGF signaling.
Although hPSC-CMs raised hopes that this human test bed could broaden drug discovery approaches and improve preclinical drug testing, they are not identical with mature adult CMs. Important distinctions in ion channel function, gene expression, structural organization and functional responses to drugs limit their application for drug testing. Compared to standard $2 \mathrm{D}$ culture formats, engineered $3 \mathrm{D}$ heart tissues improve CM maturity, and exhibit a more physiological 3D muscle environment, longitudinal alignment, and easy access of measurements of force, which is one of the most important parameters of heart function (Eder et al., 2016). However, one major drawback of EHTs is their inability to scale up for the highthroughput screening of multiple samples in parallel. To some extent, EHTs in 24-well format or cardiac tissue miniaturization might provide possible solutions for drug testing purposes (Thavandiran et al., 2013; Eder et al., 2016).

Zhao et al. (2019) developed a scalable cardiac tissue cultivation platform that reconstructs a specific anatomic portion of the heart to facilitate more directed and accurate measurements of drug responses. "Human-heart-in-a-jar" is also a related technology, which involves embedding ventricular-like hiPSC-CMs in hydrogel to create an electromechanically coupled cardiac organoids chamber that is capable of pumping fluid. This organoid chamber is amenable to clinically relevant functional measurements, such as pressure-volume loop analysis, cardiac output, and ejection fraction ( $\mathrm{Li}$ et al., 2018). As the field of iPSC technology continues to grow, to increase the efficiency of drug discovery and to reduce the cost, an established set of standards for the comparison of the quality of iPSC-CMs, quantification of cardiac contraction and electrophysiology, and validation of data and reproducibility, is desperately needed.

\section{CHALLENGES AND FUTURE OUTLOOK}

Despite major advancements in the application of PSC technology in cardiac diseases, several challenges remain, and require further in-depth research. As the structure, signaling, metabolism, and function in immature CMs are distinct from their adult counterparts, iPSC-CMs derived disease models might not be able to accurately reflect the true disease phenotype, making it difficult to confidently assess the efficacy or toxicity of drug candidates. Recently, various studies have tried to enhance the maturity of hiPSC-CMs via different means, including using T3 hormone, metabolic maturation, 3-D construction, mechanic stress, electrical stimulation, long-term culturing, and co-culture with other cell types such as cardiac fibroblasts and endothelial cells (Ahmed et al., 2020). However, it is worthwhile to point out that these "matured" cells still differ from adult human cardiomyocytes in many aspects, and therefore require careful data interpretation. Other limitations, such as scalability and clinical compatibility, also need to be addressed before one can faithfully extrapolate findings from such models. Developing optimal methods to efficiently generate large-scale mature hPSC-CMs is therefore of high importance and priority.

Furthermore, limited blood perfusion of cells within complex structures, especially for cell patches, is another issue yet to be 
overcome. Otherwise, oxygen, nutrient and drug delivery, which all depend on the microvasculature, would be compromised. Simple co-culture of cardiomyocytes with endothelial cells only generates premature micro-vasculatures that are not sufficient to support perfusion, leading to the small size of cardiac organoids (i.e., $100 \mu \mathrm{m}$ ). To introduce microvascularization into cell patches, Schaefer et al. (2018) engineered a bilayer patch composed of a layer of human iPSC-CMs and a layer of human blood outgrowth endothelial cells. Implantation of this bilayer patch into infarcted rat hearts resulted in better CM survival compared to CM-only patch control. Importantly, after 4 weeks in vivo, the engrafted microvessels sprouted into the accompanying CM layer, and even became inosculated with the host vasculature. Qian et al. (2019) co-cultured human mesenchymal stem cells and endothelial cells in decellularized human dermal fibroblast sheets. However, without actual implantation in vivo, one can hardly infer the reparative effects of such patches purely based on biochemical data. Clearly, engineering heart-like tissues with intact microvasculature is still in its infancy. Once solved, in vitro cardiac models would be able to accommodate even more diversified functional assessments, offering greater versatility and yielding higher predictability.

\section{CONCLUSION}

In this review, we outlined the current applications of hPSCCMs in disease modeling, cell therapy and drug discovery.

\section{REFERENCES}

Ahmed, R. E., Anzai, T., Chanthra, N., and Uosaki, H. (2020). A brief review of current maturation methods for human induced pluripotent stem cellsderived cardiomyocytes. Front. Cell Dev. Biol. 8:178. doi: 10.3389/fcell.2020. 00178

Bargehr, J., Ong, L. P., Colzani, M., Davaapil, H., Hofsteen, P., Bhandari, S., et al. (2019). Epicardial cells derived from human embryonic stem cells augment cardiomyocyte-driven heart regeneration. Nat. Biotechnol. 37, 895-906. doi: 10.1038/s41587-019-0197-199

Blinova, K., Dang, Q., Millard, D., Smith, G., Pierson, J., Guo, L., et al. (2018). International multisite study of human-induced pluripotent stem cell-derived cardiomyocytes for drug proarrhythmic potential assessment. Cell Rep. 24, 3582-3592. doi: 10.1016/j.celrep.2018.08.079

Breckwoldt, K., Letuffe-Brenière, D., Mannhardt, I., Schulze, T., Ulmer, B., Werner, T., et al. (2017). Differentiation of cardiomyocytes and generation of human engineered heart tissue. Nat. Protoc. 12, 1177-1197. doi: 10.1038/nprot.2017. 033

Cambria, E., Pasqualini, F. S., Wolint, P., Günter, J., Steiger, J., Bopp, A., et al. (2017). Translational cardiac stem cell therapy: advancing from first-generation to next-generation cell types. NPJ Regen. Med. 2:17. doi: 10.1038/s41536-0170024- 1

Carvajal-Vergara, X., Sevilla, A., Dsouza, S. L., Ang, Y. S., Schaniel, C., Lee, D. F., et al. (2010). Patient-specific induced pluripotent stem-cell-derived models of LEOPARD syndrome. Nature 465, 808-812. doi: 10.1038/nature09005

Caspi, O., Huber, I., Kehat, I., Habib, M., Arbel, G., Gepstein, A., et al. (2007). Transplantation of human embryonic stem cell-derived cardiomyocytes improves myocardial performance in infarcted rat hearts. J. Am. Coll. Cardiol. 50, 1884-1893. doi: 10.1016/j.jacc.2007.07.054

Chong, J. J. H., Yang, X., Don, C. W., Minami, E., Liu, Y. W., Weyers, J. J., et al. (2014). Human embryonic-stem-cell-derived cardiomyocytes regenerate non-human primate hearts. Nature 510, 273-277. doi: 10.1038/nature13233
While hPSC technology has gained momentum in cardiac repair over the years, efforts to enhance the maturity of derived cells and to increase the complexity of tissue structure are still underway. Integrating genetic-, computer-, and bioengineeringbased approaches will further empower this technique with greater precision, breadth and depth. It can be foreseen that through these interdisciplinary endeavors, hPSC technology will redefine in vitro cardiac modeling, promote personalized treatment, and increase the efficiency but reduce the cost of drug discovery.

\section{AUTHOR CONTRIBUTIONS}

All authors listed have made a substantial, direct and intellectual contribution to the work, and approved it for publication.

\section{FUNDING}

This work was supported by the National Key R\&D Program of China (2017YFA0103700), CAMS Innovation Fund for Medical Sciences (CIFMS, 2018-I2M-3-002, 2016-I2M-1-015), grants (82025004, 82088101 to LW, and 82070287 to BZ) from the National Natural Science Foundation of China, Key Laboratory of Application of Pluripotent Stem Cells in Heart Regeneration, Chinese Academy of Medical Sciences (2019PT320026), and Beijing Natural Science Foundation (Z200026).

Dell'Era, P., Benzoni, P., Crescini, E., Valle, M., Xia, E., Consiglio, A., et al. (2015). Cardiac disease modeling using induced pluripotent stem cell-derived human cardiomyocytes. World J. Stem Cells 7, 329-342. doi: 10.4252/wjsc.v7.i2.329

Eder, A., Vollert, I., Hansen, A., and Eschenhagen, T. (2016). Human engineered heart tissue as a model system for drug testing. Adv. Drug Deliv. Rev. 96, 214-224. doi: 10.1016/j.addr.2015.05.010

Fordyce, C. B., Roe, M. T., Ahmad, T., Libby, P., Borer, J. S., Hiatt, W. R., et al. (2015). Cardiovascular drug development: is it dead or just hibernating? J. Am. Coll. Cardiol. 65, 1567-1582. doi: 10.1016/j.jacc.2015.03.016

Friedrichs, S., Malan, D., and Sasse, P. (2013). Modeling long QT syndromes using induced pluripotent stem cells: current progress and future challenges. Trends Cardiovasc. Med. 23, 91-98. doi: 10.1016/j.tcm.2012.09.006

Gao, L., Gregorich, Z. R., Zhu, W., Mattapally, S., Oduk, Y., Lou, X., et al. (2018). Large cardiac muscle patches engineered from human induced-pluripotent stem cell-derived cardiac cells improve recovery from myocardial infarction in swine. Circulation 137, 1712-1730. doi: 10.1161/CIRCULATIONAHA.117. 030785

Giacomelli, E., Meraviglia, V., Campostrini, G., Cochrane, A., Cao, X., van Helden, R. W. J., et al. (2020). Human-iPSC-Derived cardiac stromal cells enhance maturation in 3D cardiac microtissues and reveal non-cardiomyocyte contributions to heart disease. Cell Stem Cell 26, 862-879.e11. doi: 10.1016/j. stem.2020.05.004

Gintant, G., Sager, P. T., and Stockbridge, N. (2016). Evolution of strategies to improve preclinical cardiac safety testing. Nat. Rev. Drug Discov. 15, 457-471. doi: $10.1038 /$ nrd.2015.34

Guo, R., Morimatsu, M., Feng, T., Lan, F., Chang, D., Wan, F., et al. (2020). Stem cell-derived cell sheet transplantation for heart tissue repair in myocardial infarction. Stem Cell Res. Ther. 11:19. doi: 10.1186/s13287-019-1536-y

Hinson, J. T., Chopra, A., Nafissi, N., Polacheck, W. J., Benson, C. C., Swist, S., et al. (2015). Titin mutations in iPS cells define sarcomere insufficiency as a cause of dilated cardiomyopathy. Science 349, 982-986. doi: 10.1126/science. aaa5458 
Hirt, M. N., Hansen, A., and Eschenhagen, T. (2014). Cardiac tissue engineering?: state of the art. Circ. Res. 114, 354-367. doi: 10.1161/CIRCRESAHA.114.30 0522

Hirt, M. N., Sörensen, N. A., Bartholdt, L. M., Boeddinghaus, J., Schaaf, S., Eder, A., et al. (2012). Increased afterload induces pathological cardiac hypertrophy: a new in vitro model. Basic Res. Cardiol. 107:307. doi: 10.1007/s00395-0120307-z

Kawamura, M., Miyagawa, S., Fukushima, S., Saito, A., Miki, K., Funakoshi, S., et al. (2017). Enhanced therapeutic effects of human iPS cell derivedcardiomyocyte by combined cell-sheets with omental flap technique in porcine ischemic cardiomyopathy model. Sci. Rep. 7:8824. doi: 10.1038/s41598-017-08 869-z

Kawamura, M., Miyagawa, S., Miki, K., Saito, A., Fukushima, S., Higuchi, T., et al. (2012). Feasibility, safety, and therapeutic efficacy of human induced pluripotent stem cell-derived cardiomyocyte sheets in a porcine ischemic cardiomyopathy model. Circulation 126, 29-37. doi: 10.1161/ CIRCULATIONAHA.111.084343

Kehat, I., Khimovich, L., Caspi, O., Gepstein, A., Shofti, R., Arbel, G., et al. (2004). Electromechanical integration of cardiomyocytes derived from human embryonic stem cells. Nat. Biotechnol. 22, 1282-1289. doi: 10.1038/nbt1014

Kim, C., Wong, J., Wen, J., Wang, S., Wang, C., Spiering, S., et al. (2013). Studying arrhythmogenic right ventricular dysplasia with patient-specific iPSCs. Nature 494, 105-110. doi: 10.1038/nature11799

Laflamme, M. A., Chen, K. Y., Naumova, A. V., Muskheli, V., Fugate, J. A., Dupras, S. K., et al. (2007). Cardiomyocytes derived from human embryonic stem cells in pro-survival factors enhance function of infarcted rat hearts. Nat. Biotechnol. 25, 1015-1024. doi: 10.1038/nbt1327

Lam, C. K., and Wu, J. C. (2018). Diseasemodelling and drug discovery for hypertrophic cardiomyopathy using pluripotent stem cells: how far have we come? Eur. Heart J. 39, 3893-3895. doi: 10.1093/eurheartj/ehy388

Laverty, H. G., Benson, C., Cartwright, E. J., Cross, M. J., Garland, C., Hammond, T., et al. (2011). How can we improve our understanding of cardiovascular safety liabilities to develop safer medicines? Br. J. Pharmacol. 163, 675-693. doi: 10.1111/j.1476-5381.2011.01255.x

Lee, J. H., Protze, S. I., Laksman, Z., Backx, P. H., and Keller, G. M. (2017). Human pluripotent stem cell-derived atrial and ventricular cardiomyocytes develop from distinct mesoderm populations. Cell Stem Cell 21, 179-194.e4. doi: 10.1016/j.stem.2017.07.003

Li, R. A., Keung, W., Cashman, T. J., Backeris, P. C., Johnson, B. V., Bardot, E. S., et al. (2018). Bioengineering an electro-mechanically functional miniature ventricular heart chamber from human pluripotent stem cells. Biomaterials 163 , 116-127. doi: 10.1016/j.biomaterials.2018.02.024

Liu, Y. W., Chen, B., Yang, X., Fugate, J. A., Kalucki, F. A., Futakuchi-Tsuchida, A., et al. (2018). Human embryonic stem cell-derived cardiomyocytes restore function in infarcted hearts of non-human primates. Nat. Biotechnol. 36, $597-$ 605. doi: $10.1038 /$ nbt. 4162

Long, C., Li, H., Tiburcy, M., Rodriguez-Caycedo, C., Kyrychenko, V., Zhou, H., et al. (2018). Correction of diverse muscular dystrophy mutations in human engineered heart muscle by single-site genome editing. Sci. Adv. 4:eaa9004. doi: 10.1126/sciadv.aap9004

Mannhardt, I., Breckwoldt, K., Letuffe-Brenière, D., Schaaf, S., Schulz, H., Neuber, C., et al. (2016). Human engineered heart tissue: analysis of contractile force. Stem Cell Rep. 7, 29-42. doi: 10.1016/j.stemcr.2016.04.011

Matsa, E., Burridge, P. W., and Wu, J. C. (2014). Human stem cells for modeling heart disease and for drug discovery. Sci. Transl. Med. 6:239s6. doi: 10.1126/ scitranslmed.3008921

Matsuura, K., Haraguchi, Y., Shimizu, T., and Okano, T. (2013). Cell sheet transplantation for heart tissue repair. J. Control. Release 169, 336-340. doi: 10.1016/j.jconrel.2013.03.003

Mercola, M., Colas, A., and Willems, E. (2013). Induced pluripotent stem cells in cardiovascular drug discovery. Circ. Res. 112, 534-548. doi: 10.1161/ CIRCRESAHA.111.250266

Mills, R. J., Parker, B. L., Quaife-Ryan, G. A., Voges, H. K., Needham, E. J., Bornot, A., et al. (2019). Drug screening in human PSC-Cardiac organoids identifies pro-proliferative compounds acting via the mevalonate pathway. Cell Stem Cell 24, 895-907.e6. doi: 10.1016/j.stem.2019.03.009

Mills, R. J., Titmarsh, D. M., Koenig, X., Parker, B. L., Ryall, J. G., Quaife-Ryan, G. A., et al. (2017). Functional screening in human cardiac organoids reveals a metabolic mechanism for cardiomyocyte cell cycle arrest. Proc. Natl. Acad. Sci. U S A. 114, E8372-E8381. doi: 10.1073/pnas.1707316114

Mummery, C. L., Zhang, J., Ng, E. S., Elliott, D. A., Elefanty, A. G., and Kamp, T. J. (2012). Differentiation of human embryonic stem cells and induced pluripotent stem cells to cardiomyocytes: a methods overview. Circ. Res. 111, 344-358. doi: 10.1161/CIRCRESAHA.110.227512

Nishiga, M., Wang, D. W., Han, Y., Lewis, D. B., and Wu, J. C. (2020). COVID-19 and cardiovascular disease: from basic mechanisms to clinical perspectives. Nat. Rev. Cardiol. 17, 543-558. doi: 10.1038/s41569-020-0413-419

Protze, S. I., Lee, J. H., and Keller, G. M. (2019). Human pluripotent stem cell-derived cardiovascular cells: from developmental biology to therapeutic applications. Cell Stem Cell 25, 311-327. doi: 10.1016/j.stem.2019.07.010

Protze, S. I., Liu, J., Nussinovitch, U., Ohana, L., Backx, P. H., Gepstein, L., et al. (2017). Sinoatrial node cardiomyocytes derived from human pluripotent cells function as a biological pacemaker. Nat. Biotechnol. 35, 56-68. doi: 10.1038/ nbt.3745

Qian, Z., Sharma, D., Jia, W., Radke, D., Kamp, T., and Zhao, F. (2019). Engineering stem cell cardiac patch with microvascular features representative of native myocardium. Theranostics 9, 2143-2157. doi: 10.7150/thno.29552

Richards, D. J., Li, Y., Kerr, C. M., Yao, J., Beeson, G. C., Coyle, R. C., et al. (2020). Human cardiac organoids for the modelling of myocardial infarction and drug cardiotoxicity. Nat. Biomed. Eng. 4, 446-462. doi: 10.1038/s41551-020-0539-4

Sager, P. T., Gintant, G., Turner, J. R., Pettit, S., and Stockbridge, N. (2014). Rechanneling the cardiac proarrhythmia safety paradigm: a meeting report from the cardiac safety research consortium. Am. Heart J. 167, 292-300. doi: 10.1016/j.ahj.2013.11.004

Savoji, H., Mohammadi, M. H., Rafatian, N., Toroghi, M. K., Wang, E. Y., Zhao, Y. et al. (2019). Cardiovascular disease models: a game changing paradigm in drug discovery and screening. Biomaterials 198, 3-26. doi: 10.1016/j.biomaterials. 2018.09.036

Schaefer, J. A., Guzman, P. A., Riemenschneider, S. B., Kamp, T. J., and Tranquillo, R. T. (2018). A cardiac patch from aligned microvessel and cardiomyocyte patches. J. Tissue Eng. Regen. Med. 12, 546-556. doi: 10.1002/term.2568

Seeger, T., Porteus, M., and Wu, J. C. (2017). Genome editing in cardiovascular biology. Circ. Res. 120, 778-780. doi: 10.1161/CIRCRESAHA.116.310197

Sharma, A., Burridge, P. W., McKeithan, W. L., Serrano, R., Shukla, P., Sayed, N., et al. (2017). High-throughput screening of tyrosine kinase inhibitor cardiotoxicity with human induced pluripotent stem cells. Sci. Transl. Med. 9:eaaf2584. doi: 10.1126/scitranslmed.aaf2584

Sharma, A., McKeithan, W. L., Serrano, R., Kitani, T., Burridge, P. W., del, et al. (2018). Use of human induced pluripotent stem cell-derived cardiomyocytes to assess drug cardiotoxicity. Nat. Protoc. 13, 3018-3041. doi: 10.1038/s41596018-0076-78

Sharma, A., Sances, S., Workman, M. J., and Svendsen, C. N. (2020). Multi-lineage human iPSC-Derived platforms for disease modeling and drug discovery. Cell Stem Cell 26, 309-329. doi: 10.1016/j.stem.2020.02.011

Tachibana, A., Santoso, M. R., Mahmoudi, M., Shukla, P., Wang, L., Bennett, M., et al. (2017). Paracrine effects of the pluripotent stem cell-derived cardiac myocytes salvage the injured myocardium. Circ. Res. 121, e22-e36. doi: 10.1161/ CIRCRESAHA.117.310803

Te Riele, A. S. J. M., Agullo-Pascual, E., James, C. A., Leo-Macias, A., Cerrone, M., Zhang, M., et al. (2017). Multilevel analyses of SCN5A mutations in arrhythmogenic right ventricular dysplasia/cardiomyopathy suggest noncanonical mechanisms for disease pathogenesis. Cardiovasc. Res. 113, 102-111. doi: $10.1093 / \mathrm{cvr} / \mathrm{cvw} 234$

Thavandiran, N., Dubois, N., Mikryukov, A., Massé, S., Beca, B., Simmons, C. A., et al. (2013). Design and formulation of functional pluripotent stem cellderived cardiac microtissues. Proc. Natl. Acad. Sci. U. S. A. 110, E4698-E4707. doi: 10.1073/pnas. 1311120110

Theodoris, C. V., Zhou, P., Liu, L., Zhang, Y., Nishino, T., Huang, Y., et al. (2020). Network-based screen in iPSC-derived cells reveals therapeutic candidate for heart valve disease. Science 371:eabd0724. doi: 10.1126/science.abd0724

Tzatzalos, E., Abilez, O. J., Shukla, P., and Wu, J. C. (2016). Engineered heart tissues and induced pluripotent stem cells: macro- and microstructures for disease modeling, drug screening, and translational studies. Adv. Drug Deliv. Rev. 96, 234-244. doi: 10.1016/j.addr.2015.09.010

Voges, H. K., Mills, R. J., Elliott, D. A., Parton, R. G., Porrello, E. R., and Hudson, J. E. (2017). Development of a human cardiac organoid injury model reveals 
innate regenerative potential. Development 144, 1118-1127. doi: 10.1242/dev. 143966

Wang, G., McCain, M. L., Yang, L., He, A., Pasqualini, F. S., Agarwal, A., et al. (2014). Modeling the mitochondrial cardiomyopathy of barth syndrome with induced pluripotent stem cell and heart-on-chip technologies. Nat. Med. 20, 616-623. doi: 10.1038/nm.3545

Weng, Z., Kong, C. W., Ren, L., Karakikes, I., Geng, L., He, J., et al. (2014). A simple, cost-effective but highly efficient system for deriving ventricular cardiomyocytes from human pluripotent stem cells. Stem Cells Dev. 23, 17041716. doi: $10.1089 / \mathrm{scd} .2013 .0509$

Williams, I. M., and Wu, J. C. (2019). Generation of endothelial cells from human pluripotent stem cells methods, considerations, and applications. Arterioscler. Thromb. Vasc. Biol. 39, 1317-1329. doi: 10.1161/ATVBAHA.119. 312265

Yang, L., Han, Y., Nilsson-Payant, B. E., Gupta, V., Wang, P., Duan, X., et al. (2020). A human pluripotent stem cell-based platform to study SARS-CoV-2 tropism and model virus infection in human cells and organoids. Cell Stem Cell 27, 125-136.e7. doi: 10.1016/j.stem.2020.06.015

Yazawa, M., Hsueh, B., Jia, X., Pasca, A. M., Bernstein, J. A., Hallmayer, J., et al. (2011). Using induced pluripotent stem cells to investigate cardiac phenotypes in timothy syndrome. Nature 471, 230-236. doi: 10.1038/nature09855

Ye, L., Chang, Y. H., Xiong, Q., Zhang, P., Zhang, L., Somasundaram, P., et al. (2014). Cardiac repair in a porcine model of acute myocardial infarction with human induced pluripotent stem cell-derived cardiovascular cells. Cell Stem Cell. 15, 750-761. doi: 10.1016/j.stem.2014.11.009

Yiangou, L., Davis, R. P., and Mummery, C. L. (2020). Using cardiovascular cells from human pluripotent stem cells for COVID- 19 research: why the heart fails. Stem Cell Rep. 16, 385-397. doi: 10.1016/j.stemcr.2020.11.003

Zhang, B., Montgomery, M., Chamberlain, M. D., Ogawa, S., Korolj, A., Pahnke, A., et al. (2016a). Biodegradable scaffold with built-in vasculature for organ-on-a-chip engineering and direct surgical anastomosis. Nat. Mater. 15, 669-678. doi: 10.1038/nmat4570

Zhang, J., Tao, R., Campbell, K. F., Carvalho, J. L., Ruiz, E. C., Kim, G. C., et al. (2019). Functional cardiac fibroblasts derived from human pluripotent stem cells via second heart field progenitors. Nat. Commun. 10:2238. doi: 10.1038/ s41467-019-09831-9835

Zhang, Y. S., Aleman, J., Shin, S. R., Kilic, T., Kim, D., Shaegh, S. A. M., et al. (2017). Multisensor-integrated organs-on-chips platform for automated and continual in situ monitoring of organoid behaviors. Proc. Natl. Acad. Sci. U S A. 114, E2293-E2302. doi: 10.1073/pnas.1612906114

Zhao, Y., Rafatian, N., Feric, N. T., Cox, B. J., Aschar-Sobbi, R., Wang, E. Y., et al. (2019). A platform for generation of chamber-specific cardiac tissues and disease modeling. Cell 176, 913-927.e18. doi: 10.1016/j.cell.2018.11.042

Zhao, Y., Rafatian, N., Wang, E. Y., Wu, Q., Lai, B. F. L., Lu, R. X., et al. (2020). Towards chamber specific heart-on-a-chip for drug testing applications. $A d v$. Drug Deliv. Rev. 165-166, 60-76. doi: 10.1016/j.addr.2019.12.002

Zheng, Y. Y., Ma, Y. T., Zhang, J. Y., and Xie, X. (2020). COVID-19 and the cardiovascular system. Nat. Rev. Cardiol. 17, 259-260. doi: 10.1038/s41569020-0360-365

Conflict of Interest: The authors declare that the research was conducted in the absence of any commercial or financial relationships that could be construed as a potential conflict of interest.

Copyright (c) 2021 Huang, Feng, Wang and Zhou. This is an open-access article distributed under the terms of the Creative Commons Attribution License (CC BY). The use, distribution or reproduction in other forums is permitted, provided the original author(s) and the copyright owner(s) are credited and that the original publication in this journal is cited, in accordance with accepted academic practice. No use, distribution or reproduction is permitted which does not comply with these terms. 